\title{
Environmental Function Evaluation and Preparation of Green Decorative Materials in Indoor Design
}

\author{
Jing Liang \\ Department of Art and Design, Shijiazhuang University of Applied Technology, Shijiazhuang 050081, China
}

Corresponding Author Email: xiaoyao_11520@126.com

https://doi.org/10.18280/acsm.450509

Received: 5 July 2021

Accepted: 1 September 2021

Keywords:
green decorative materials, indoor design,
environmental function evaluation,
preparation of wood product coating

\section{Keywords:}

environmental function evaluation,

preparation of wood product coating

\begin{abstract}
With the growing environmental awareness, there is a rising demand for green decorative materials in furniture and indoor design. However, the existing studies lack the overall evaluation of environmental functions for green decorative materials in indoor design. To make up for the gap, this paper evaluates the environmental function and explores the preparation method of green decorative materials in indoor design. Firstly, the types and purchase principles of green decorative materials were expounded, and the preparation flow of wood product coating was given for green decorative materials in indoor design. Then, an evaluation model was established for the environmental functions of green decorative materials, the stochastic dominance matrix was constructed for the indices, and the order value of each index was computed. Finally, fuzzy Dempster-Shafer (D-S) evidence theory was adopted to develop a prediction algorithm for the environmental function of indoor decorations, and thus realize the evaluation of the greenness of decorative materials in indoor design. Experimental results demonstrate the effectiveness of our algorithm, and the good performance of the prepared super-hydrophobic and oleophobic coatings.
\end{abstract}

\section{INTRODUCTION}

As a result of social-economic integration, personalized interior design not only brings comfort and beauty, but also seriously pollutes the indoor environment. In future, consumers will pursue greener, more environmentallyfriendly, and more efficient decorative materials for indoor design [1-4]. The greening of indoor decorations helps to improve the living environment and the health condition [5-7]. Visually speaking, green indoor decorative materials can improve the mood of the occupants, and even relieve fatigue [8-15]. With the growing awareness of health, there is a rising demand for green decorative materials in furniture and indoor design [16-19]. In recent years, various green decorative materials with diverse functions have been researched and developed. This trend testifies the surge in the demand for indoor green decorative materials.

Ling [20] noted that the functions and environmental friendliness of indoor decorative nanomaterials are the current research hotspot, carried out three survey experiments on the role of decorative nanomaterials in indoor design and the application of environmental protection concepts, and compared the environmental parameters of traditional decorative materials and those of green nanomaterials. The experimental results show that indoor decorative design with green nanomaterials reduces the emissions of harmful and toxic materials. Taking a construction project as the object, $\mathrm{Lu}$ [21] monitored the gaseous pollution (e.g., hydrogen sulfide and sulfur dioxide), solid pollution, water pollution, light pollution and noise pollution sources in the construction site, and demonstrated that high-quality construction of green buildings can be realized through overall design of energy conservation and environmental protection, the structural optimization design, and heating, ventilation, and air conditioning (HVAC) design of green buildings. Wang and $\mathrm{Li}$ [22] combined high-performance phase change composites with the system of energy-saving green indoor decorative materials into a temperature-automatically controlled energyefficient green decorative material system based on phase change composites. Ryder [23] pointed out the development trend of modern building materials (traditional building materials cannot meet people's needs, while new environmentally friendly building materials can satisfy our demand), and detailed the new green construction materials and their applications. $\mathrm{Li}$ [24] described a series of commercial processes for indoor design coatings, which are pollution-free, low-cost, fast to apply, and user friendly, with a small capital/equipment cost.

The existing studies lack the overall evaluation of environmental functions for green decorative materials in indoor design. This paper probes deep into the functions, roles, and preparation of green decorative materials from the following aspects: the development of green decorative materials, the different types of green decorative materials, the environmental functions of green decorative materials in indoor space, and the limitations and prospects of the application of green decorative materials. Starting from the current state of household-based indoor green decorative materials in modern cities, this paper mainly involves the following contents: Section 2 expounds the types and purchase principles of green decorative materials, and specifies the preparation flow of wood product coating for green decorative materials in indoor design. Section 3 establishes an evaluation model for the environmental functions of green decorative 
materials, constructs the stochastic dominance matrix for the indices, and computes the order value of each index. Section 4 adopts fuzzy Dempster-Shafer (D-S) evidence theory to develop a prediction algorithm for the environmental function of indoor decorations, and thus realize the evaluation of the greenness of decorative materials in indoor design. Experimental results demonstrate the effectiveness of our algorithm, and the good performance of the prepared superhydrophobic and oleophobic coatings.

\section{TYPES AND PURCHASE PRINCIPLES OF GREEN DECORATIVE MATERIALS}

Common green decorative materials include soft stone floor, integrated wallboard, green wall facing, latex paint, diatomaceous earth, etc. The stone floor, which is mixed from natural marble powder and other polymers, boasts a rich texture, a good quality, as well as high greenness, energyefficiency, and recyclability. Currently, the popular integrated wallboards are mainly made of nanofibers, bamboo fibers and Al-Mn alloy. These materials have advantages in terms of insulation, fire prevention, moisture-proof, environmental performance, wear resistance, and convenient installation. The grass wallpaper and hemp wallpaper in the market have good moisturizing, anti-mildew and peeling effects. These two green decorative materials win the favor of consumers for their rich colors and beautiful appearance. Latex paint is aqueous decorative paint, with a certain moisture-proof and antimildew effect. This beautiful and scrub-resistant product has been widely used on interior walls. Diatomaceous earth is also favored in indoor decorative design, owing to its excellent environmental performance, flame retardancy, sound absorption ability, and good air purification effect.

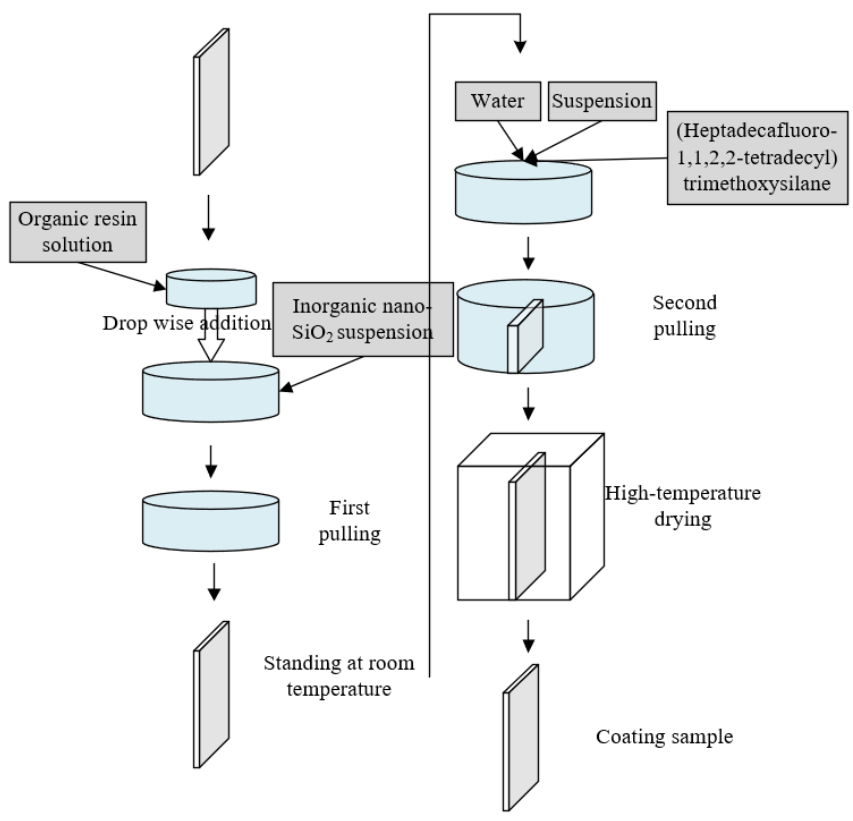

Figure 1. Preparation flow of wood product coating for green indoor decoration

During the purchase of green decorative materials, the walls should be decorated with wooden boards as much as possible, applied with pollution-free polyvinyl chloride (PVC) green wallpapers, or painted with smooth aqueous coatings. The preferred floor material is pollution-resistant and nonradioactive natural stones. If solid wood flooring is adopted, the glue must have low organic emissions. The wood products need to be coated with green coatings. Never use odorous benzene-containing diluents that volatile rapidly.

Wood product coatings are shifting from solvent coating to green coating. Green aqueous coatings are less harmful to the human body than powder coatings and curable coatings. This paper explains the preparation flow of a super-hydrophobic and oleophobic coating for green indoor decorations (Figure 1), aiming to transcend the constraint of drying condition on coating production, reduce the use of highly toxic organic solvents, and improve the durability and aesthetics of the coating material.

Indoor decoration is directly associated with the health and safety of consumers, and concerned by all walks of life. Strictly speaking, it is difficult to clearly define whether a green decorative material is to be applied indoor, outdoor, or in special venues. Therefore, this paper, focusing on green indoor decorative materials, firstly evaluates the environmental functions of green decorative materials, and then discusses the preparation technique of the superhydrophobic and oleophobic coating.

\section{MODEL CONSTRUCTION}

\subsection{Stochastic dominance matrix}

Let $R_{i}$ be type i green decorative material. Then, the set of $\mathrm{u}$ types of green decorative materials can be denoted as $R=\left\{R_{1}, R_{2}, \ldots, R_{i}, \ldots, R_{n}\right\}$. Let $F Z_{j}$ be an evaluation index of the environmental functions of green decorative materials. Then, the set of $\mathrm{m}$ such indices can be denoted as $F Z=\left\{F Z_{1}, F Z_{2}, \ldots, F Z_{i}, \ldots, F Z_{m}\right\}$. Based on the calculation of greenness, the environmental function levels of an index $F Z_{j}$ can be determined as $\psi^{\tau}=\left\{\psi^{1}=1, \psi^{2}=2, \psi^{3}=3, \psi^{4}=4, \psi^{5}=5\right\}$.

The stochastic dominance between the indices $F Z_{1}, F Z_{2}, \ldots$, $F Z_{l}, \ldots, F Z_{m}$ can be determined as follows: Firstly, the cumulative probability distribution functions (CPDFs) of all random variables, which bear on the values of the evaluation indices, are compared randomly by the optimal allocation principle of stochastic dominance. Then, the all indices can be ranked by superiority/inferiority.

To begin with, it is necessary to compute the expectation $p_{S}$ of the CPDF $G_{S}(\varphi)$ of the environmental function levels corresponding to the greenness scores of green decorative material $R_{i}$ on all indices. Contraposing expert comment set $D$, the number of expert comments on green decorative material $R_{i}$, which assign the environmental function on index $F Z_{j}$ to level $\psi^{\tau}$, is denoted as $C O^{\tau}{ }_{i j}$. According to $C O_{i j}$ and $C O^{\tau}{ }_{i j}$, the probability for the environmental function of green decorative material $R_{i}$ on index $F Z_{j}$ to fall on level $\psi^{\tau}$ can be calculated by:

$$
\underset{\operatorname{pro}_{i j}}{\stackrel{C}{C O_{i j}}}
$$

Let $G_{i j}(\varphi)$ be the CPDF of environmental function of green decorative material $R_{i}$ on index $F Z_{j} ; G_{i f}(\varphi)$ be the CPDF of the environmental function of green decorative material $R_{i}$ on index $F Z_{f}$ falling on level $\psi^{\tau}$. When $G_{i j}(\varphi)$ has stochastic dominance over $G_{i f}(\varphi), F Z_{j}$ must have stochastic dominance over $F Z_{f}$. The CPDF of the environmental function of green 
decorative material $R_{i}$ on index $F Z_{j}$ falling on level $\psi^{\tau}$ can be given by:

$$
G_{i j}(\phi)=\sum_{\phi \geq \psi^{\tau}} \underset{i j}{\stackrel{\tau}{\operatorname{rro}^{2}}}
$$

On this basis, it is possible to construct the expected vector $P_{i}=\left(p_{i j}\right)_{i * 1}$ for the environmental function level of green decorative material $R_{i}$ on index $F Z_{j}$, where $p_{i j}$ can be defined as:

$$
p_{i j}(\phi)=\sum_{\tau=1}^{5} \operatorname{Pro} \psi_{i j}^{\tau}
$$

Next, it is necessary to construct the stochastic dominance matrix $s_{j f}^{i}$ between any two indices of green decorative material $R_{i}$. The comments of all experts are converted into the probability distributions of the environmental function levels of the green decorative material on the indices. After that, any two indices of green decorative material $R_{i}$ are compared by the stochastic dominance rule. The stochastic dominance $S_{i}=\left(s_{j f}^{i}\right)_{M z t \times M s t}$ between indices $F Z_{j}$ and $F Z_{f}$ of green decorative material $R_{i}$ can be characterized by:

$$
s_{j f}^{i}=\left\{\begin{array}{l}
F O R, G_{i j}\left(\psi^{\tau}\right) \text { FORG }_{i f}\left(\psi^{\tau}\right) \\
\operatorname{SOR}, G_{i j}\left(\psi^{\tau}\right) \operatorname{SORG}_{i f}\left(\psi^{\tau}\right) \\
\operatorname{TOR}, G_{i j}\left(\psi^{\tau}\right) \operatorname{TORG}_{i f}\left(\psi^{\tau}\right) \\
/, \text { Otherwise }
\end{array}\right.
$$

where, $s_{j f}^{i}=F O R$ means index $F Z_{j}$ of green decorative material $R_{i}$ has first-order stochastic dominance over $F Z_{f}, s_{j f}^{i}=S O R$ means index $F Z_{j}$ of green decorative material $R_{i}$ has secondorder stochastic dominance over $F Z_{f}, s_{j f}^{i}=T O R$ means index $F Z_{j}$ of green decorative material $R_{i}$ has third- order stochastic dominance over $F Z_{f}, s_{j f}^{i_{j}}=/$ means stochastic dominance does not exist between indices $F Z_{j}$ and $F Z_{f}$.

\subsection{Oder value of indices}

(1) Matrix of stochastic dominance degrees

Based on the constructed stochastic dominance matrix $S_{i}=\left(s_{j f}\right)_{M z t \times M s t}$, an order function is established by hesitant fuzzy method to further depict the stochastic dominance degree between any two indices $F Z_{j}$ and $F Z_{f}$ of green decorative material $R_{i}$. Then, the overall order matrix is set up through pairwise comparison of indices to compute the order value of each index. On this basis, the indices of the green decorative material can be ranked. Let $a_{i} \beta_{i}$ be the preference threshold of green decorative material $R_{i}$. Then, the matrix of stochastic dominance degrees $R D_{i}=\left[r d_{i}\left(F Z_{j}, F Z_{f}\right)\right]_{M \times N}$ can be derived by:

$$
r d_{i}\left(F Z_{j}, F Z_{f}\right)=\left\{\begin{array}{l}
1, r_{i h}^{j}=S D, \text { and } p_{i j} \geq p_{i f}+\beta_{i} \\
\frac{p_{i j}-p_{i f}}{\beta_{i}}, s_{i f}^{j}=S D, \text { and } p_{i f}<p_{i j}<p_{i j}+\beta_{i} \\
0, \text { Otherwise }
\end{array}\right.
$$

$\beta_{i}$ can be calculated by:

$$
\begin{aligned}
& \beta_{i}=\frac{2}{m(m-1)} \sum_{j=1}^{m} \sum_{f=1 f \neq j}^{m} \xi_{j f}^{i}(m=1,2, \ldots, 21 ; f=1,2, . ., 21) \\
& \text { where, } \xi_{j f}^{i}=\left\{\begin{array}{l}
p_{i j}-p_{i f}, p_{i j} \geq p_{i f} \\
0, p_{i j}<p_{i f}
\end{array}\right.
\end{aligned}
$$

For $R D_{i}=\left[r d_{i}\left(F Z_{j}, F Z_{f}\right)\right]_{m \times m}$, the dominance of $F Z_{j}$ over $F Z_{f}$ is positively correlated with the value of $r d_{i}\left(F Z_{j}, F Z_{f}\right)$.

(2) Order value of each index

To compute the order value $\delta_{i}\left(F Z_{j}\right)$ for each index of each green decorative material, the first step is to calculate the outflow $\delta^{+}{ }_{i}\left(F Z_{j}\right)$ and inflow $\delta_{i}{ }_{i}\left(F Z_{j}\right)$ of index $F Z_{l}$ of green decorative material $R_{i}$, based on the stochastic dominance matrix $R D_{i}=\left[r d_{i}\left(F Z_{j}, F Z_{f}\right)\right]_{m \times m}$. Note that $\delta^{+}{ }_{i}\left(F Z_{j}\right)$ and $\delta_{i}{ }_{i}\left(F Z_{j}\right)$ are the confidence of $F Z_{j}$ being superior and inferior to the other index, respectively. The greater the $\delta^{+}{ }_{i}\left(F Z_{j}\right)$, the smaller the $\delta_{i}^{-}\left(F Z_{j}\right)$, and the better the environmental functions of the material on the corresponding index. According to the matrix of stochastic dominance degree $R D_{i}=\left[r d_{i}\left(F Z_{j}, F Z_{f}\right)\right]_{m \times m}, \delta^{+}{ }_{i}\left(F Z_{j}\right)$ and $\delta_{i}^{-}\left(F Z_{j}\right)$ can be respectively defined by:

$$
\begin{aligned}
& \delta_{i}^{+}\left(F Z_{j}\right)=\sum_{m} r d\left(F Z_{j}, F Z_{f}\right) \\
& \delta_{i}^{-}\left(F Z_{j}\right)=\sum_{m} r d\left(F Z_{f}, F Z_{j}\right)
\end{aligned}
$$

According to $\delta^{+}{ }_{i}\left(F Z_{j}\right)$ and $\delta_{i}^{-}\left(F Z_{j}\right)$, the order value $\delta_{i}\left(F Z_{j}\right)$ of each index of each green decorative material can be calculated by:

$$
\delta_{i}\left(F Z_{j}\right)=\delta_{i}^{+}\left(F Z_{j}\right)-\delta_{i}^{-}\left(F Z_{j}\right)
$$

The greater the order value $\delta_{i}\left(F Z_{j}\right)$, the better the environmental functions of the material on index $F Z_{j}$.

\section{EVALUATION OF GREEN ENVIRONMENTAL FUNCTIONS}

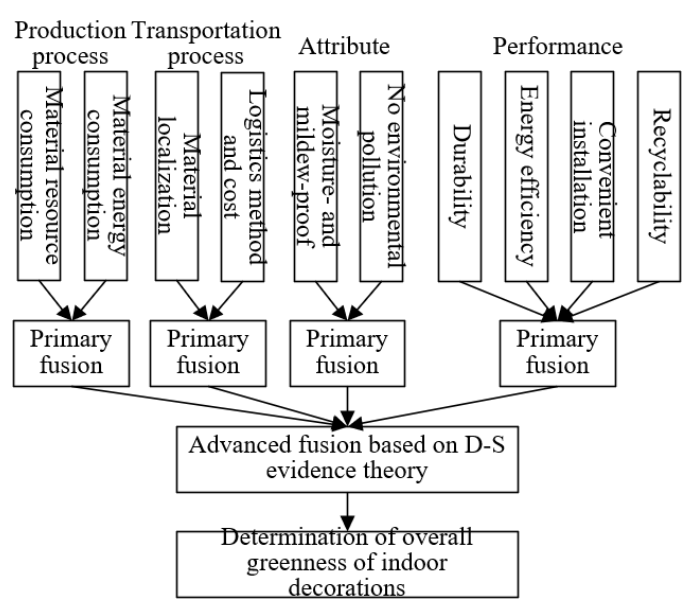

Figure 2. Flow of evaluation model

Various types of decorative materials are adopted for indoor design. To accurately evaluate the overall greenness of indoor decorations, it is necessary to integrate the evaluation information of environmental functions of each decorative material by a certain optimization rule, and further process the 
fused information. Considering the floating error of the relevant indices, as well as the weight difference between indices, this paper proposes an environmental function prediction algorithm for indoor decorations based on fuzzy D$\mathrm{S}$ evidence theory. Then, the greenness of indoor design decorations was evaluated based on the index data acquired previously. Figure 2 shows the flow of the evaluation model for the greenness of indoor designs.

Relying on data intelligence algorithm, this paper processes the fused information on the environmental functions of different decorative materials. The index data of $\mathrm{m}$ green decorative materials at time $\mathrm{h}$ can be obtained from the definition of the optimal fuzzy set at time h. For this set, the environmental function information of green decorative materials $R_{i}$ and $R_{j}$ at time $\mathrm{h}$ is denoted as $E_{i}(h)$ and $E_{j}(h)$, respectively. The values of $E_{i}(h)$ and $E_{j}(h)$ are negatively correlated with the complexity of the fusion between index data of the two materials at time $h$.

To facilitate the analysis of the fusion complexity between index data, this paper introduces the fusion membership function $W_{i j}(h) \in[0, \mathrm{k}]$, which can be generated through the mapping between $E_{i}(h)$ and $E_{j}(h)$. The value of membership function $W_{i j}(h)$ can be characterized by the degree of fusion between the index data of any two green decorative materials at time h. The membership function $W_{i j}(h)$ can be defined as:

$$
W_{i j}(h)=\exp \left\{-\frac{1}{2}\left|e_{i}(h)-e_{j}(h)\right|\right\}
$$

The closer the $W_{i j}(h)$ value is to 1 , the deeper the fusion between the index data of two green decorative materials; the closer the $W_{i j}(h)$ value is to 0 , the shallower the fusion. Based on the degree of fusion, the data fusion matrix $W$ can be established as:

$$
W=\left[\begin{array}{cccc}
1 & W_{12}(h) & \cdots & W_{1 n}(h) \\
W_{21}(h) & 1 & \cdots & W_{2 n}(h) \\
\vdots & \vdots & \ddots & \ddots \\
W_{n 1}(h) & W_{n 2}(h) & \cdots & 1
\end{array}\right]
$$

If the sum of the elements in any row of matrix $W$ is large, there is a small gap between $E_{i}(h)$ and the mean; if the sum is small, there is a large deviation of the index data on green decorative material $R_{i}$. The consistent fusion degree of the indices for material $R_{i}$ can be expressed as:

$$
\lambda_{i}(h)=\sum_{j=1}^{n} w_{i j}(h) / n
$$

However, the mean consistent fusion degree calculated by formula (12) cannot reflect the stability of environmental functions of green decorative materials. If the index data on green decorative material $R_{i}$ remain stable with the elapse of time, the fusion degree of the data will deviate greatly from that of the evaluation information of other green decorative materials, and exert a major impact on the uniform distribution of fusion degrees. The distribution balance of green decorative material $R_{i}$ at time h can be expressed as:

$$
\gamma_{i}(h)=1 / \sum_{j=1}^{n}\left(v_{i}(h)-w_{i j}(h)^{2}\right) / n
$$

During indoor design, preference should be given to green decorative materials with consistent, and uniformly distributed fusion degrees. The distribution and weight of fusion degree depend on the uniformity and fusion coefficient of green decorative materials. Hence, the fusion degree weights can be balanced with the uniform fusion degree coefficient and distribution balance coefficient. The weight coefficient of green decorative material $R_{i}$ at time h can be calculated by:

$$
\theta_{i}(h)=\lambda_{i}(h) \times \gamma_{i}(h)
$$

The result of formula (14) can be normalized by:

$$
\theta_{i}(h)=\Phi(h) / \sum_{i=1}^{n} \Phi_{i}(h)
$$

The final fusion result can be given by:

$$
\hat{a}=\sum_{i=1}^{n} \theta_{i}(h) e_{i}(h)=\sum_{i=1}^{n} \frac{\theta_{i}(h) e_{i}(h)}{\sum_{i=1}^{n} \theta_{i}(h)}
$$

The D-S evidence theory can overcome the limits of prior probability and the corresponding conditional probability. By applying this theory to the evaluation of the overall greenness of multiple indoor green decorative materials, the environmental function information of these materials can be described through the reasoning and decision-making processes of D-S evidence.

The conflicting decisions are greatly affected by lowreliable evidence. To minimize the effect, this paper proposes a consistent fusion algorithm for the reliability of conflicting evidence, which excels in modification based on the consistency of original reliable evidence, and modification based on the consistency of combination rules. The proposed algorithm simultaneously enhances the evidence reliability of environmental function information on green decorative materials, and the accuracy of evidence fusion based on the information, reducing the influence of reliable evidence weighting on composite decision. Let $n_{1}$ and $n_{2}$ be the basic confidence distribution functions; $X_{i}$ and $Y_{i}$ be the focal elements. The basic probability distribution function can be expressed as:

$$
\begin{aligned}
& n(X)=0, X=\varphi \\
& n(X)=\frac{\sum_{X_{i} Y_{i}=X} n_{1}\left(X_{i}\right) n_{2}\left(Y_{j}\right)}{1-\sum_{X_{i} Y_{i}=\delta} n_{1}\left(X_{i}\right) n_{2}\left(Y_{j}\right)}, X=\varphi
\end{aligned}
$$

In the evaluation data fusion system, the global reliability of each evidence can be given by:

$$
\lambda_{l}\left(Q_{l}\right)=\max _{1 \leq i \leq n}\left(\lambda_{i}\left(Q_{i}\right)\right)
$$

The weight evidence in the system, i.e., the most reliable evidence in the calculation results, is called the weight evidence $\lambda_{l}$ of the fusion system. Each weight is assigned a weight referring to the global reliability of the evidence by:

$$
\gamma_{i}=\frac{\lambda_{i}\left(Q_{i}\right)}{\lambda_{l}\left(Q_{l}\right)}=\frac{\lambda_{i}\left(Q_{i}\right)}{\max _{1 \leq i \leq n} \lambda_{i}\left(Q_{i}\right)}
$$


The basic reliability $R E_{i}(h)$ can be obtained through normalization:

$$
R E_{i}(h)=\Phi_{i}(h) / \sum_{i=1}^{n} \Phi_{i}(h)
$$

By reassigning the weight coefficients to evidence, the basic probability distribution function can be obtained as:

$$
n_{i}\left(X_{l}\right)=\left\{\begin{array}{l}
\gamma_{i} \bullet n_{i}\left(X_{l}\right) \\
1-\sum \gamma_{i} \bullet n_{i}\left(Y_{l}\right)
\end{array}\right.
$$

The new basic probability distribution function (21) can be fused by the following combination rules:

$$
\left\{\begin{array}{l}
n(\varsigma)=0 \\
n(X)=\sum_{\substack{X_{l} \cap Y_{l}=X \\
X_{l} \bullet Y_{l} \subseteq \Psi}} \tilde{n}_{i}\left(X_{l}\right) \bullet \tilde{n}_{j}\left(Y_{l}\right)+\sum_{\substack{X_{l} \cap Y_{l}=\varphi \\
Y_{l} \subseteq \Psi^{\prime}}} \mu\left(X, Y_{l}\right)
\end{array}\right.
$$

With $\gamma_{i}=1$, a new mass function can be obtained as:

$$
n_{i}\left(\tilde{X}_{l}\right)=\left\{\begin{array}{l}
n_{i}\left(X_{l}\right) \\
1-\sum n_{i}\left(Y_{l}\right)
\end{array}\right.
$$

Substituting ten indices $R_{1}-R_{10}$ (material resource consumption; material energy consumption; material localization; logistics method and cost; moisture- and mildewproof; no environmental pollution; durability; energy efficiency; convenient installation; recyclability) into formula (23), the basic probability distribution function can be obtained as:

$$
\tilde{n}(A)\left\{\begin{array}{l}
n\left(\alpha_{1}\right) \ldots A=\alpha_{1} \\
n\left(\alpha_{2}\right) \ldots A=\alpha_{2} \\
n\left(\alpha_{3}\right) \ldots A=\alpha_{3} \\
n\left(\alpha_{4}\right) \ldots A=\alpha_{4} \\
n\left(\alpha_{5}\right) \ldots A=\alpha_{5} \\
1-n\left(\alpha_{1}\right)-n\left(\alpha_{2}\right)-n\left(\alpha_{3}\right)-n\left(\alpha_{4}\right)-n\left(\alpha_{5}\right) \ldots A=\Psi
\end{array}\right.
$$

The consistency can be described as:

$$
\mu\left(X, Y_{l}\right)=\frac{\tilde{n}_{i}\left(X_{l}\right)^{3} \tilde{n}_{j}(X)}{\tilde{n}_{i}(X)^{2}+\tilde{n}_{j}\left(Y_{l}\right)^{2}}+\frac{\tilde{n}_{i}\left(Y_{l}\right)^{3} \tilde{n}_{j}(X)}{\tilde{n}_{i}\left(Y_{l}\right)^{2}+\tilde{n}_{j}(X)^{2}}
$$

Formula (25) shows that the proposed algorithm fully mines the information consistency between highly reliable evidence, and effectively solves the conflicts between environmental function information and evidence. In addition, each evidence is weighted through full consideration of the safety and reliability of evaluation information.

\section{EXPERIMENTS AND RESULTS ANALYSIS}

Table 1 lists the outflow, inflow, and order value of the environmental functions of green decorative materials. The order value $\delta$ is positively correlated with the environmental function level of the corresponding green decorative material

\begin{tabular}{|c|c|c|c|c|c|c|c|c|c|c|}
\hline Index & $F Z_{1}$ & $F Z_{2}$ & $F Z_{3}$ & $F Z_{4}$ & $F Z_{5}$ & $F Z_{6}$ & $F Z_{7}$ & $F Z_{8}$ & $F Z_{9}$ & $F Z_{10}$ \\
\hline Material 1 & 0.685 & 0.658 & 0.594 & 0.623 & 0.584 & 0.625 & 0.594 & 0.612 & 0.572 & 0.615 \\
\hline Material 2 & 0.724 & 0.602 & 0.715 & 0.630 & 0.185 & 0.802 & 0.736 & 0.632 & 0.285 & 0.826 \\
\hline Result & 0.785 & 0.652 & 0.534 & 0.032 & 0.745 & 0.716 & 0.685 & 0.695 & 0.623 & 0.258 \\
\hline Index & $F Z_{1}^{\prime}$ & $F Z_{2}^{\prime}$ & $F Z^{\prime}{ }_{3}$ & $F Z^{\prime}{ }_{4}$ & $F Z^{\prime}{ }_{5}$ & $F Z_{6}^{\prime}$ & $F Z^{\prime}{ }_{7}$ & $F Z_{8}^{\prime}$ & $F Z^{\prime}{ }_{9}$ & $F Z^{\prime}{ }_{10}$ \\
\hline Material 1 & 0.625 & 0.658 & 0.524 & 0.613 & 0.564 & 0.525 & 0.614 & 0.635 & 0.537 & 0.524 \\
\hline Material 2 & 0.714 & 0.637 & 0.712 & 0.627 & 0.143 & 0.826 & 0.748 & 0.615 & 0.238 & 0.827 \\
\hline Result & 0.736 & 0.637 & 0.548 & 0.048 & 0.772 & 0.728 & 0.638 & 0.649 & 0.638 & 0.273 \\
\hline
\end{tabular}
on index $F Z_{j}$. Thus, the evaluation indices can be ranked by the order value.

Table 1. Outflow, inflow, and order value of each index

\begin{tabular}{ccccccccccc}
\hline Index & $\boldsymbol{F Z}_{\mathbf{1}}$ & $\boldsymbol{F Z}_{\mathbf{2}}$ & $\boldsymbol{F Z}_{\mathbf{3}}$ & $\boldsymbol{F Z}_{\mathbf{4}}$ & $\boldsymbol{F Z}_{\mathbf{5}}$ & $\boldsymbol{F Z}_{\mathbf{6}}$ & $\boldsymbol{F Z}_{\mathbf{7}}$ & $\boldsymbol{F Z}_{\mathbf{8}}$ & $\boldsymbol{F Z}_{\boldsymbol{9}}$ & $\boldsymbol{F Z}_{\mathbf{1 0}}$ \\
\hline$\delta^{+}$ & 3.2564 & 1.625 & 0.824 & 6.321 & -5.326 & -6.251 & -7.235 & -3.625 & -0.008 & -9.254 \\
$\delta^{-}$ & 3.7856 & 1.652 & 0.852 & 6.233 & -5.324 & -6.258 & -7.253 & -3.625 & -0.018 & -9.254 \\
$\delta$ & 3.7584 & 1.625 & 0.825 & 6.235 & -5.125 & -6.352 & -7.251 & -3.256 & -0.007 & -9.021 \\
\hline Index & $\boldsymbol{F Z}_{\mathbf{1}}^{\prime}$ & $\boldsymbol{F Z}_{\mathbf{2}}$ & $\boldsymbol{F Z}_{\mathbf{3}}$ & $\boldsymbol{F Z}_{\mathbf{4}}$ & $\boldsymbol{F Z}_{\mathbf{5}}$ & $\boldsymbol{F Z}_{\mathbf{6}}$ & $\boldsymbol{F Z}_{\boldsymbol{7}}$ & $\boldsymbol{F Z}_{\mathbf{8}}$ & $\boldsymbol{F Z}_{\mathbf{9}}^{\prime}$ & $\boldsymbol{F Z}_{\mathbf{1 0}}$ \\
\hline$\delta^{+}$ & -4.2525 & -4.613 & -3.825 & -4.313 & -3.324 & -6.221 & -8.215 & 4.614 & 4.034 & 2.2254 \\
$\delta^{-}$ & -4.7836 & -4.652 & -8.824 & -4.238 & -5.334 & -6.214 & -4.248 & 3.652 & 4.035 & 2.285 \\
$\delta$ & -3.7258 & -4.614 & -5.818 & -4.259 & -5.127 & -6.319 & -7.237 & 4.248 & 4.018 & 2.019 \\
\hline
\end{tabular}

Table 2. Fused environmental function information of different green decorative materials

Table 3. Coating transmittance at different first pulling rates

\begin{tabular}{ccccccc}
\hline & Blank glass & $\mathbf{1 . 0}$ & $\mathbf{2 . 0}$ & $\mathbf{3 . 0}$ & $\mathbf{4 . 0}$ & $\mathbf{5 . 0}$ \\
\hline Maximum & 91.35 & 85.27 & 79.26 & 82.15 & 78.62 & 79.28 \\
Minimum & 88.21 & 82.16 & 80.26 & 76.29 & 73.26 & 69.28 \\
Mean & 90.25 & 83.26 & 82.29 & 79.29 & 77.26 & 75.29 \\
Standard deviation & 0.82 & 0.46 & 0.85 & 1.62 & 1.79 & 2.95 \\
\hline
\end{tabular}


Table 2 shows the fused environmental function information of different green decorative materials. Materials 1 and 2 were unqualified in terms of production process, and qualified on the other aspects. Before the experiments, an expert survey was carried out. The results indicate that the environmental functions of both materials are unqualified. Thus, the results of our algorithm are inconsistent with the results of expert survey. The proposed evaluation model achieved an accuracy of $97 \%$ in the environmental function evaluation of green decorative materials. This is of certain practical significance, as it meets the application standard for household-based indoor design.

The high performance of the prepared super-hydrophobic and oleophobic coating was verified through contrastive experiments. Tables 3 and 4 present the coating transmittance at different first and second pulling rates. The pulling rate was set to $1.0 \mathrm{~cm} / \mathrm{s}, 2.0 \mathrm{~cm} / \mathrm{s}, 3.0 \mathrm{~cm} / \mathrm{s}, 4.0 \mathrm{~cm} / \mathrm{s}$, and $5.0 \mathrm{~cm} / \mathrm{s}$, in turn. As shown in Table 3, the transmittance of the flat coating gradually decreased with the growth of first pulling rate. When the first pulling rate was below $2.0 \mathrm{~cm} / \mathrm{s}$, the mean transmittance of the prepared coating was above 0.8 in the range of visible light wavelengths; When the first pulling rate was above $2.0 \mathrm{~cm} / \mathrm{s}$, the mean transmittance of the prepared coating was smaller than 0.8 in the range of visible light wavelengths. Hence, the transmittance of the superhydrophobic and oleophobic coating is superior at a low first pulling rate.

As shown in Table 4, within the range of visible light wavelengths, the coating transmittance did not fluctuate significantly, as the second pulling rate changed from $1.0 \mathrm{~cm} / \mathrm{s}$, $2.0 \mathrm{~cm} / \mathrm{s}, 3.0 \mathrm{~cm} / \mathrm{s}, 4.0 \mathrm{~cm} / \mathrm{s}$, to $5.0 \mathrm{~cm} / \mathrm{s}$. In the short wavelength range, the transmittance quickly declined until 0 . Besides, the mean transmittance did not drop significantly, with the growth of second pulling rate, and the standard deviation remained small. Therefore, the transmittance of the prepared superhydrophobic and oleophobic coating is not greatly affected by second pulling rate. Figure 3 shows the composite performance of the prepared coating. Obviously, our coating achieved the best overall performance at the second pulling rate of $1.0 \mathrm{~cm} / \mathrm{s}$.

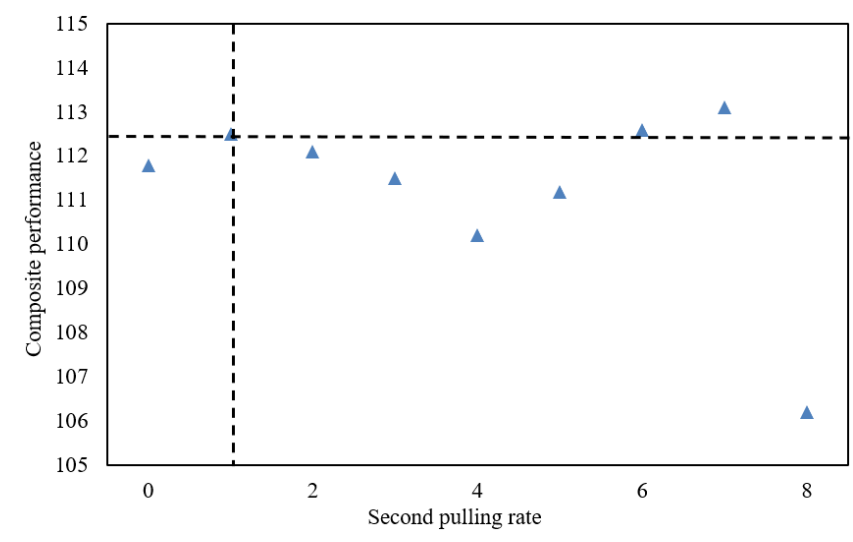

Figure 3. Composite performance of the prepared coating

The super-hydrophobic and oleophobic coating was prepared with nontoxic or low-toxic solvents. Table 5 shows the physical properties of the coating prepared from aminopropylene resin. Before second pulling, the suspension was prepared with four solvents: isopropanol, n-ethane, acetone, and valinone. The coating prepared with the first three solvents were not super-hydrophobic, while that prepared with isopropanol and acetone was both hydrophobic and oleophobic; that prepared with valinone was the only coating realizing super-hydrophobicity and oleophobicity, but the transmittance of the coating was merely $80.59 \%$. Table 6 shows the physical properties of the coating prepared from polysiloxane resin. Before second pulling, the suspension was prepared with four solvents: isopropanol, n-ethane, acetone, and valinone. The coatings prepared with all four solvents achieved super-hydrophobicity and oleophobicity. The transmittance of the coating prepared with acetone was only $67.9 \%$, while that of the coating prepared with any of the other solvents surpassed $85 \%$.

Table 4. Coating transmittance at different second pulling rates

\begin{tabular}{ccccccc}
\hline & Blank glass & $\mathbf{1 . 0}$ & $\mathbf{2 . 0}$ & $\mathbf{3 . 0}$ & $\mathbf{4 . 0}$ & $\mathbf{5 . 0}$ \\
\hline Maximum & 92.36 & 89.23 & 77.29 & 83.17 & 79.64 & 77.25 \\
Minimum & 89.24 & 86.15 & 81.24 & 78.25 & 74.24 & 73.26 \\
Mean & 92.37 & 87.25 & 88.25 & 77.32 & 79.24 & 72.25 \\
Standard deviation & 0.85 & 0.49 & 0.82 & 1.66 & 1.73 & 2.97 \\
\hline
\end{tabular}

Table 5. Physical properties of the coating prepared from aminopropylene resin

\begin{tabular}{ccccc}
\hline & Isopropanol & n-ethane & Acetone & Valinone \\
\hline Water contact Angle (\%) & 142.8 & 125.3 & 138.5 & 155.8 \\
Oil contact Angle (\%) & 105.6 & 80.3 & 102.5 & 108.4 \\
Transmittance (\%) & 58.6 & 85.2 & 85.9 & 80.59 \\
Fog degree (\%) & 92.35 & 48.25 & 82.16 & 85.29 \\
Adhesion (\%) & 100 & 101 & 100 & 101 \\
\hline
\end{tabular}

Table 6. Physical properties of the coating prepared from polysiloxane resin

\begin{tabular}{ccccc}
\hline & Isopropanol & n-ethane & Acetone & Valinone \\
\hline Water contact Angle (\%) & 153.6 & 127.4 & 135.7 & 156.6 \\
Oil contact Angle (\%) & 106.4 & 81.5 & 103.4 & 109.6 \\
Transmittance (\%) & 67.9 & 86.4 & 86.7 & 85.54 \\
Fog degree (\%) & 93.38 & 47.24 & 81.15 & 87.27 \\
Adhesion (\%) & 101 & 100 & 100 & 100 \\
\hline
\end{tabular}




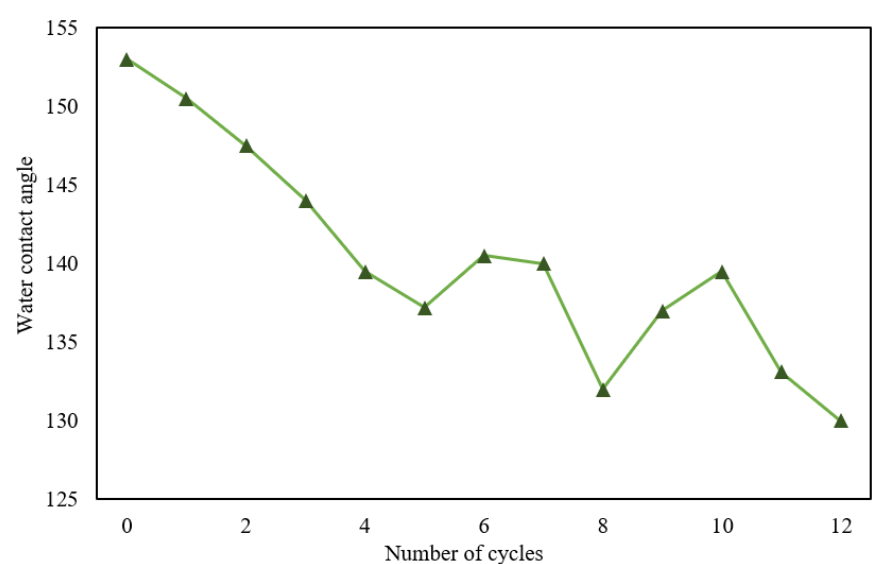

(1)

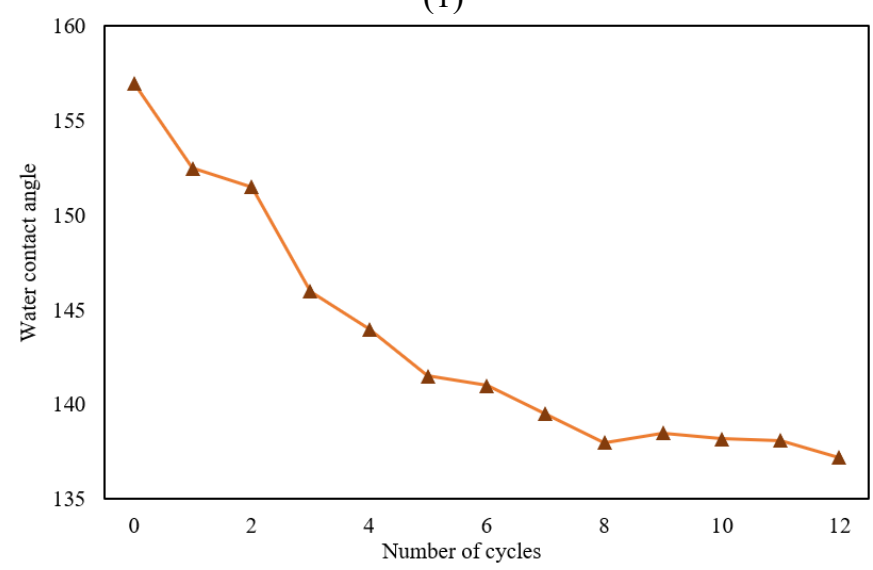

(2)

Figure 4. Wear resistance of the prepared coating

Figure 4 shows the test results on the wear resistance of the coatings prepared with aminopropylene resin and polysiloxane resin. The wear resistance test was carried out in the following steps: Place the super-hydrophobic and oleophobic coating sample with an additional $80 \mathrm{~g}$ weight evenly on a piece of sandpaper, and make the sample move forward at uniform speed by $15 \mathrm{~cm}$ against the friction of the sandpaper. Four flips of the sample were treated as a cycle. Figure 4 shows the change of water contact angle of the sample after each cycle. It can be seen that the water contact angle of the two coatings both reduced with the growing number of cycles. After 8 cycles, the water contact angle of the coating prepared with polysiloxane resin gradually stabilized, while that of the coating prepared with aminopropylene resin fluctuated. This means the coating prepared with polysiloxane resin is relatively stable and good in performance.

\section{CONCLUSIONS}

This paper evaluates the environmental function and explores the preparation method of green decorative materials in indoor design. Firstly, the authors explained the types and purchase principles of green decorative materials, and clarified the preparation flow of wood product coating for green decorative materials in indoor design. Then, an evaluation model was established for the environmental functions of green decorative materials, and a prediction algorithm was developed for the environmental function of indoor decorations based on fuzzy D-S evidence theory, laying the basis for evaluating the greenness of decorative materials in indoor design. Through experiments, the fused environmental protection information was obtained for different green decorative materials, which demonstrates the effectiveness of our evaluation method. Further, the coating transmittance was counted at different first and second pulling rates, and the composite performance of the coatings was plotted. It was concluded that the super-hydrophobic and oleophobic coatings have a relatively good transmittance at a low first pulling rate, and their transmittance is not greatly affected by second pulling rate. Finally, the physical properties and wear resistance of the prepared super-hydrophobic and oleophobic coatings were tested. The test results confirm the superior performance of the prepared coatings.

\section{REFERENCES}

[1] Yu, W., Tang, S., Wang, K. (2021). Research on energysaving performance of low-energy consumption green buildings. E3S Web of Conferences, Les Ulis, 233: 01100. https://doi.org/10.1051/e3sconf/202123301100

[2] Yin, Q., Shi, X., Xiong, S., Qu, Y., Zhou, Y., Liu, J., Jia, Z., Ma, L. (2021). Selection of water-saving plants and annual water consumption estimation for garden green spaces in Beijing. Forests, 12(11): 1572. https://doi.org/10.3390/f12111572

[3] Xue, F., Zhao, J. (2021). Application calibration based on energy consumption model in optimal design of green buildings. Advances in Materials Science and Engineering, 2021: 5360443. https://doi.org/10.1155/2021/5360443

[4] Goyal, M., Goyal, R.K. (2021). Confirming antecedents of green consumption intention: A sustainable model for food aggregators. IETE Journal of Research, 67(5): 634645. https://doi.org/10.1080/03772063.2021.1906336

[5] Wu, Z., Li, H., Feng, Y., Luo, X., Chen, Q. (2019). Developing a green building evaluation standard for interior decoration: A case study of China. Building and Environment, 152: 50-58. https://doi.org/10.1016/j.buildenv.2019.02.010

[6] Tian, G., Zhang, H., Feng, Y., Wang, D., Peng, Y., Jia, H. (2018). Green decoration materials selection under interior environment characteristics: A grey-correlation based hybrid MCDM method. Renewable and Sustainable Energy Reviews, 81: 682-692. https://doi.org/10.1016/j.rser.2017.08.050

[7] Zhou, M. (2013). On interior greening decoration of residence. Applied Mechanics and Materials, 275: 27932796.

https://doi.org/10.4028/www.scientific.net/AMM.275277.2793

[8] Yavaşer, R., Karagözler, A.A. (2020). Reactive green 5decorated polyacrylamide/chitosan cryogel: An affinity matrix for catalase. Applied Biochemistry and Biotechnology, 192(4): 1191-1206. https://doi.org/10.1007/s12010-020-03393-9

[9] Perera, M., Wijenayaka, L.A., Siriwardana, K., Dahanayake, D., de Silva, K.N. (2020). Gold nanoparticle decorated titania for sustainable environmental remediation: Green synthesis, enhanced surface adsorption and synergistic photocatalysis. RSC Advances, 10(49): 29594-29602. https://doi.org/10.1039/D0RA05607C

[10] Pazoki, F., Bagheri, S., Shamsayei, M., Nejad, M.J., Heydari, A. (2020). BiPO4 decorated with Ni-Fe layered 
double hydroxide as a highly efficient and reusable heterogeneous catalyst for aldol condensation in green solvent. Materials Chemistry and Physics, 253: 123327. https://doi.org/10.1016/j.matchemphys.2020.123327

[11] Iranmanesh, T., Foroughi, M.M., Jahani, S., Zandi, M.S., Nadiki, H.H. (2020). Green and facile microwave solvent-free synthesis of $\mathrm{CeO}_{2}$ nanoparticle-decorated CNTs as a quadruplet electrochemical platform for ultrasensitive and simultaneous detection of ascorbic acid, dopamine, uric acid and acetaminophen. Talanta, 207:

120318. https://doi.org/10.1016/j.talanta.2019.120318

[12] Gadallah, M.I., Ali, H.R.H., Askal, H.F., Saleh, G.A. (2019). Poly (bromocresol green) flakes-decorated pencil graphite electrode for selective electrochemical sensing applications and pharmacokinetic studies. Materials Science and Engineering: C, 102: 634-645. https://doi.org/10.1016/j.msec.2019.03.071

[13] Gao, M., Wang, Z., Yang, C., Ning, J., Zhou, Z., Li, G. (2019). Novel magnetic graphene oxide decorated with persimmon tannins for efficient adsorption of malachite green from aqueous solutions. Colloids and Surfaces A: Physicochemical and Engineering Aspects, 566: 48-57. https://doi.org/10.1016/j.colsurfa.2019.01.016

[14] Sharifuzzaman, M., Barman, S.C., Zahed, M.A., San, N. J., Park, J.Y. (2019). Green synthesis of reduced graphene oxide decorated with few-layered MoS2nanoroses and $\mathrm{Au} / \mathrm{Pd} / \mathrm{Ag}$ trimetallic nanoparticles for ultrasensitive label-free immunosensing platforms. Journal of The Electrochemical Society, 166(4): B249B257. https://doi.org/10.1149/2.0861904jes

[15] Xu, J., Gu, P., Birch, D.J., Chen, Y. (2018). Plasmonpromoted electrochemical oxygen evolution catalysis from gold decorated $\mathrm{MnO} 2$ nanosheets under green light. Advanced Functional Materials, 28(31): 1801573. https://doi.org/10.1002/adfm.201801573

[16] Hariez, A., Taha, A.A., Wenshui, X. (2019). Simple and green synthesis of mesoporous nanofibers decorated by Ag-nanoparticles and their application as an antibacterial membrane. Advances in Natural Sciences: Nanoscience and Nanotechnology, 10(3): 035002. http://dx.doi.org/10.1088/2043-6254/ab2e6d
[17] Sadhukhan, S., Ghosh, T.K., Roy, I., Rana, D., Bhattacharyya, A., Saha, R., Chattopadhyay, S., Khatua, S., Acharya, K., Chattopadhyay, D. (2019). Green synthesis of cadmium oxide decorated reduced graphene oxide nanocomposites and its electrical and antibacterial properties. Materials Science and Engineering: C, 99: 696-709. https://doi.org/10.1016/j.msec.2019.01.128

[18] Palai, P.K., Mondal, A., Chakraborti, C.K., Banerjee, I., Pal, K. (2019). Green synthesized amino-PEGylated silver decorated graphene nanoplatform as a tumortargeted controlled drug delivery system. SN Applied Sciences, 1(3): 269. https://doi.org/10.1007/s42452-0190287-9

[19] Su, X. (2021). Function of nano-decorative materials in interior design and the application of ecological and environmental protection concepts. Ferroelectrics, 580(1): 268-282. https://doi.org/10.1080/00150193.2021.1902774

[20] Ling, X.S. (2021). Application of energy saving and environmental protection green decoration materials in building construction. Forest Chemicals Review, 46-52. https://doi.org/10.17762/jfcr.vi.49

[21] Lu, X. (2020). Research on the application of green energy-saving and environmental protection decoration materials in building decoration construction. International Journal of Environmental Technology and Management, 23(2-4): 233-244. https://dx.doi.org/10.1504/IJETM.2020.112966

[22] Wang, A., Li, M.M. (2016). Discussion on the development and application of new environmental protection building materials. Proceedings of the 3rd Annual International Conference on Material Science and Engineering, ICMSE 2015, Guangdong, China, pp. 481-484.

[23] Ryder, P. (1999). Decorative/protective electrophoretic coatings-an environmentally friendly alternative to electroplating \& other finishes. AESF SUR FINProceedings, pp. 487-488.

[24] Li, X. (2017). Application of energy conservation and environmental protection measures in construction of building decoration. Chemical Engineering Transactions, 62: 511-516. https://doi.org/10.3303/CET1762086 